PharmacoEconomics \& Outcomes News 865, p31 - 31 Oct 2020

\title{
The PACTS principles for patient involvement in health economics research
}

The development of principles for patient involvement in health economics research offers "useful foundations" for economists to use alongside existing approaches to public and patient involvement (PPI), according to an article published in The Patient.

The UK-based researchers drew on their 7-year experience working with patients with multiple sclerosis to offer guidance on the 'patient involvement' component of PPI in health economics research. The resultant set of principles is titled PACTS: Planning; Approach selection; Continuous involvement; Team building; and Sensitivity.

In terms of the first component, planning, the authors found it was most beneficial to build in patient involvement to overall research plans at the application stage, including clear communication of the background and reasons for involvement, as well as specific goal setting for involvement activities. The planning component also needs to be responsive to changing circumstances, such as the COVID-19 pandemic or significant changes in the treatment landscape.

Approach selection refers to the two main approaches to involvement, namely discussion-based and task-based involvement. The authors found that discussion-based approaches were beneficial for generating wider insights, unearthing "unknown unknowns" and providing critical information from patients with lived experience of a condition. In comparison, task-based approaches provided a useful framework for more focussed methods of investigating "known unknowns" related to specific aspects of a study. The appropriateness of the different approaches depends on the nature and purpose of a particular research activity, with the authors noting that "this is not an 'either-or' dichotomy, and that the two approaches can be used in concert". They suggested that the division of sessions into discussion- and task-based segments offers a useful balance of information and insights.

The continuous involvement of patients throughout the research process and across different projects contributes to building patient expertise as well as insight for researchers, with the involvement of patients in developing patient involvement being a crucial factor. This also lends itself to the fourth component, team building, as meaningful involvement was found to create a sense of shared ownership of the research and worked towards creation of a team ethos over time.

Lastly, the importance of sensitivity was highlighted to counteract the perception of health economics as a technical or impersonal field of research. Consideration of patient needs, as well as adoption of an open, honest approach, were found to be key to developing working relationships with patients over time.

"There is increased recognition that patients are experts at providing a 'lived experience' perspective in HE [health economics] research", concluded the authors. "We hope the PACTS Principles . . . will ultimately build towards the development of practical guidance for patient involvement in HE research".

Hawton A, et al. Involving Patients in Health Economics Research: "The PACTS Principles". The Patient - Patient-Centered Outcomes Research : 12 Oct 2020. Available from: URL: https://doi.org/10.1007/s40271-020-00461-4 